\title{
GROUP DUALITY AND ISOMORPHISMS OF FOURIER AND FOURIER-STIELTJES ALGEBRAS FROM A $W^{*}$-ALGEBRA POINT OF VIEW
}

\author{
BY MARTIN E. WALTER ${ }^{1}$
}

\author{
Communicated by Irving Glicksberg, March 20, 1970
}

0. Introduction. In this paper two commutative, semisimple Banach algebras with involution, viz., $A(G)$ and $B(G)$, (called the Fourier algebra of $G$ and the Fourier-Stieltjes algebra of $G$, respectively) are defined (as in [5]) for an arbitrary locally compact topological group $G$. [Note. As algebras $A(G)$ and $B(G)$ are subalgebras of the algebra of bounded continuous functions on $G$.] The main purpose of this paper is to show that these algebras deeply reflect the structure of $G$ even though they apparently are "trivial" in the sense that multiplication is defined pointwise-not by convolution as, for example, in the case of $L^{1}(G)$.

The first main result is closely related to a well-known duality theorem, cf. [4], [5], [9], [10], [13], and it has a relatively short proof.

The group $G$ is recovered topologically as a special subset of the spectrum of $B(G)$ (or as the spectrum of $A(G)$ ), and by virtue of the fact that the dual of $B(G)$ or of $A(G)$, denoted $B(G)^{\prime}, A(G)^{\prime}$ respectively, can be naturally equipped with an algebraic structure, $G$ can be recovered algebraically as well. The main difference between our Theorem 1 and the above results is that we proceed directly to recovering the group $G$ in the $B(G), B(G)^{\prime}$ context and deduce the duality theorem in the $A(G), A(G)^{\prime}$ context as an easy corollary; whereas the other papers attack the $A(G), A(G)^{\prime}$ duality first and, except for [4] and a long complicated formulation in [13], do not discuss the process of recovering the group from the $B(G), B(G)^{\prime}$ duality. We also obtain the formula in Theorem 1 (i) and further clarify the relationship between $B(G)$ and the representation theory of $G$.

AMS 1970 subject classifications. Primary 22D25, 22D35; Secondary 22D10, 4300, 43A10.

Key words and phrases. Topological group, unitary representation, tensor product of representations, Fourier-algebra, Fourier-Stieltjes algebra, $C^{*}$-algebra of a group, $W^{*}$-algebra of a group, spectrum, group duality, isometric, isomorphism.

1 The author gratefully acknowledges the financial support given him as a National Science Foundation Fellow and the moral and mathematical support provided by Dr. B. Russo and Dr. M. Takesaki with whom the author has had several enjoyable and informative conversations. This research forms a portion of the author's Ph.D. thesis. 
The second main result is that two locally compact groups $G_{1}$ and $G_{2}$ are topologically isomorphic iff $B\left(G_{1}\right)$ is isometrically isomorphic to $B\left(G_{2}\right)$ (or iff $A\left(G_{1}\right)$ is isometrically isomorphic to $A\left(G_{2}\right)$ ). For example, consider the special case where $G_{1}$ and $G_{2}$ are abelian locally compact groups. $B\left(G_{i}\right)$ is then the Fourier-Stieltjes transform of $M^{1}\left(\hat{G}_{i}\right)$, where $\hat{G}_{i}$ is the dual group of $G_{i}$, and if $u$ is the FourierStieltjes transform of a $\mu \in M^{\prime}\left(\hat{G}_{i}\right),\|u\|_{B\left(G_{i}\right)}=\|\mu\|_{1}(i=1,2)$. Our theorem then states that any isometric isomorphism of $B\left(G_{1}\right)$ onto $B\left(G_{2}\right)$ is induced by a bicontinuous isomorphism of $G_{2}$ onto $G_{1}$, followed by a translation of $G_{1}$. In particular, $G_{1}$ is topologically isomorphic to $G_{2}$. As it turns out, even in the general case, where $G_{1}$ and $G_{2}$ are nonabelian, we can conclude that $G_{1}$ is either topologically isomorphic or topologically anti-isomorphic to $G_{2}$. If a topological anti-isomorphism from $G_{1}$ onto $G_{2}$ is followed by $g \in G_{2} \mapsto g^{-1}$, the composition is a topological isomorphism. [Note. This result is analogous to a result of Wendel [15], which states $G_{1}$ is topologically isomorphic to $G_{2}$ iff $L^{1}\left(G_{1}\right)$ is isometrically isomorphic to $L^{1}\left(G_{2}\right)$. Our result is perhaps a little more surprising, since multiplication in $L^{1}(G)$ is chosen to closely reflect the structure of $G$. For example, $L^{1}(G)$ is abelian iff $G$ is abelian.]

1. Let $G$ be a locally compact topological group. A continuous function $\varphi: G \rightarrow C$ (C designates the complex numbers) is of positive type iff for any $g_{1}, g_{2}, \cdots, g_{n}$ in $G$ the matrix $\left(\varphi\left(g_{i}^{-1} g_{j}\right)\right) 1 \leqq i, j \leqq n$ is positive hermitian. Let $P(G)$ be the set of all continuous functions of positive type on $G$. As an algebra let $B(G)$ be the set of all complex linear combinations of functions from $P(G)$. As in [5] $B(G)$ can be given a norm making it in to a commutative, semisimple Banach algebra. $B(G)$ also has two isometric involutions, viz., $b(g)=(b(g))^{-}$ and $\tilde{b}(g)=\left(b\left(g^{-1}\right)\right)$ - where $b \in B(G), g \in G$ and-indicates complex conjugation. [Note. From the theory of Banach algebras [8, Corollary (2.5.18) ], we see that the norm topology on $B(G)$ is unique. Thus the group $G$ directly dictates a unique norm topology for $B(G)$.] With this norm $B(G)$ is canonically isometric with the dual of $C^{*}(G)$, the group $C^{*}$-algebra of $G$, cf. $[2,13.9 .1$.]. Thus the dual of $B(G)$ is identified with the double dual of $C^{*}(G)$, which is a $W^{*}$-algebra denoted by $W^{*}(G)$, cf. $[2, \S 12]$. Let $W^{*}(G)_{u}$ denote the unitary operators in $W^{*}(G)$. There exists a bicontinuous isomorphism $\omega: G \rightarrow \omega(G)$ $\subset W^{*}(G)_{u}$, where $W^{*}(G)_{u}$ is given the relativized $\sigma\left(W^{*}(G), B(G)\right.$ ) topology, henceforth referred to as the $\sigma$-weak topology. After identifying $G$ and $\omega(G)$ two facts of fundamental importance are: 
(i) every continuous unitary representation $\pi$ of $G$ on a Hilbert space $H_{\pi}$ extends uniquely to a normal homomorphism $\tilde{\pi}$ of $W^{*}(G)$ in to the bounded linear operators on $H_{\pi}$, and

(ii) the $W^{*}(G), B(G)$ duality preserves the original relationship between $B(G)$ and $G$, i.e., $\langle b, \omega(g)\rangle=b(g)$ for $b \in B(G)$, and $g \in G$, cf. [5].

The Fourier algebra $A(G)$ may be defined as $\left\{f * \tilde{g}: f, g \in L^{2}(G)\right\}$ where $*$ denotes convolution. It can be shown that $A(G)$ is a norm closed ideal in $B(G)$, and the dual of $A(G)$ can be canonically identified with the von Neumann algebra generated by the left regular representation of $G$. This algebra will be denoted as $M(G)$. As a matter of notation we will denote the spectrum of $A(G)$ by $\sigma(A(G)$ ) (and the spectrum of $B(G)$ by $\sigma(B(G))$ ). For notations and definitions regarding the tensor product of representations and operators see [1, Chapter $1,2.3$ and 2.4] as well as $[2,13.1 .5$.]. A single' will be used to denote the dual of a given normed linear space. We can now state the first main result.

Theorem 1. $B(G)$ is a commutative, semisimple Banach algebra with a unit and with involution such that $B(G)^{\prime}=W^{*}(G)$ and

(i) $\sigma(B(G)) \cap W^{*}(G)_{u} \cong G$, where $\cong$ indicates a bicontinuous isomorphism.

(ii) $\sigma(B(G))=\left\{A \in W^{*}(G)-\{0\}:\left(\pi_{1} \otimes \pi_{2}\right)^{\sim}(A)=\tilde{\pi}_{1}(A) \otimes \tilde{\pi}_{2}(A)\right.$, where $\pi_{1}, \pi_{2}$ vary over all continuous unitary representations of $\left.G\right\}$ $=\left\{A \in W^{*}(G)-\{0\}:(\omega \otimes \omega)^{\sim}(A)=\tilde{\omega}(A) \otimes \tilde{\omega}(A), \omega\right.$ the universal representation of $G\}$.

(iii) $\sigma\left(B(G)\right.$ ) is a $\sigma$-weakly compact, self adjoint subset of $\left(W^{*}\right)_{1}$ $=\left\{A \in W^{*}(G):\|A\|_{w^{*}=1}\right\}$ and $X, Y \in \sigma(B(G)) \Rightarrow X Y \in \sigma(B(G)) \cup\{0\}$.

(iv) $G \subset \sigma(B(G))$ is such that $G \cong \sigma(A(G))$ and $G^{c} \cap \sigma(B(G)) \subset A(G)^{\circ}$, where ${ }^{\circ}$ indicates the polar in the $W^{*}(G), B(G)$ duality, and $G^{c}$ indicates the set theoretic complement of $G$ in $W^{*}(G)$.

REMARK. The key lemma of the proof is: The hull of $A(G)$ in $\sigma(B(G))$ is $G^{c} \cap \sigma(B(G))$, i.e., if $M \in \sigma(B(G)) \cap G^{c}$ and $I_{M}$ is the kernel of $M$, then $I_{M} \supset A(G)$.

REMARK. In (i), $W^{*}(G)_{u}$ can be replaced by $W^{*}(G)_{r}$, the set of invertible elements in $W^{*}(G)$.

REMARK. Several known results follow easily from this theorem, cf. [14]. The second main result is:

THEOREM 2. Let $G_{1}, G_{2}$ be locally compact groups. Suppose that there exists an isometric isomorphism $\phi$ of $B\left(G_{1}\right)$ onto $B\left(G_{2}\right)$. Then there 
exists (canonically) either a topological isomorphism $\psi_{I}$ of $G_{2}$ onto $G_{1}$ or a topological anti-isomorphism $\psi_{A}$ of $G_{2}$ onto $G_{1}$ and a $g_{0} \in G_{1}$ such that either

$$
\phi(b)(g)=b\left(g_{0} \psi_{I}(g)\right), \quad b \in B\left(G_{1}\right), \quad g \in G_{2}
$$

or

$$
\phi(b)(g)=b\left(g_{0} \psi_{A}(g)\right), \quad b \in B\left(G_{1}\right), \quad g \in G_{2} .
$$

The following theorem was essentially first conjectured by $M$. Takesaki.

THEOREM $2^{\prime}$. Theorem 2 remains true if $B\left(G_{i}\right)$ is replaced by $A\left(G_{i}\right)$, $i=1,2$.

REMARK. As is often times the case, the most difficult part of the proof of either of the last two theorems is the isolation of the essential "kernels of proof". "Kernel one" is the application of the Jacobson, Rickart, Kadison results as found in [6]. "Kernel two" surprisingly enough is the simple observation that the elements of $G$ are linearly independent in $W^{*}(G)$, (or $M(G)$ ), cf. [3].

CoROLLARY. If $G_{1}$ and $G_{2}$ are locally compact topological groups, then

$$
G_{1} \cong G_{2} \text { iff } B\left(G_{1}\right) \cong B\left(G_{2}\right) \text { and } G_{1} \cong G_{2} \text { iff } A\left(G_{1}\right) \cong A\left(G_{2}\right) \text {, }
$$

where

$\cong$ between groups designates a bicontinuous isomorphism, and

$\cong$ between Banach algebras designates an isometric isomorphism.

REMARK. In the course of investigation, interesting properties of the $W^{*}(G), B(G)$ duality were found. For a more detailed account of this duality, and the $M(G), A(G)$ duality, and for complete proofs to Theorems 1, 2, 2' (and other related results) see [14].

\section{REFERENCES}

1. J. Dixmier, Les algebres d'operateurs dans l'espace hilbertien (Algebres de von Neumann), Cahiers Scientifiques, fasc. 25, Gauthier-Villars, Paris, 1957. MR 20 \#1234.

2. - Les $C^{*}$-algèbres et leurs representations, Cahiers Scientifiques, fasc. 29, Gauthier-Villars, Paris, 1964. MR 30 \#1404.

3. J. Ernest, $A$ new group algebra for locally compact groups, Amer. J. Math. 86 (1964), 467-492. MR 29 \#4838.

4. - Hopf-von Neumann algebras, Proc. Conference Functional Analysis (Irvine, Calif., 1966) Academic Press, New York, 1967, pp. 195-215. MR 36 \#6956.

5. P. Eymard, L'algèbra de Fourier d'un groupe localement compact, Bull. Soc. Math. France 92 (1964), 181-236. MR 37 \#4208.

6. R. V. Kadison, Isometries of operator algebras, Ann. of Math. (2) 54 (1951), 325-338. MR 13, 256. 
7. Y. Misonou, On the direct product of $W^{*}$-algebras, Tôhoku Math. J. 6 (1954), 189-204. MR 16, 1125.

8. C. E. Rickart, General theory of Banach algebras, University Series in Higher Math., Van Nostrand, Princeton, N. J., 1960. MR 22 \#5903.

9. K. Saito, On a duality for locally compact groups, Tohoku Math. J. (2) 20 (1968), 355-367. MR 39 \#357.

10. W. F. Stinespring, Integration theorems for gages and duality for unimodular groups, Trans. Amer. Math. Soc. 90 (1959), 15-56. MR 21 \#1547.

11. Michio Suzuki, Structure of a group and the structure of its lattice of subgroups, Ergebnisse Math., Heft 10, Springer-Verlag, Berlin, 1956. MR 18, 715.

12. M. Takesaki, A characterization of group algebras as a converse of TannakaStinespring-Tatsuuma duality theorem, Amer. J. Math. 91 (1969), 529-564. MR 39 \#5752.

13. N. Tatsuuma, A duality theorem for locally compact groups, J. Math. Kyoto Univ. 6 (1967), 187-293. MR 36 \#313.

14. M. E. Walter, $W^{*}$-algebras and non-abelian harmonic analysis, (to appear).

15. J. G. Wendel, Left centralizers and isomorphisms of group algebras, Pacific J. Math. 2 (1952), 251-261. MR 14, 246.

University of California, Irvine, California 92664 